\title{
Facettes et reflets du mythe mirandolien ${ }^{1}$
}

LOUIS

VALCKE

Résumé: Il existe autour de l'Oratio de hominis dignitate de Jean Pic de la Mirandole un véritable mythe prométhéen, par lequel le texte de l'Oratio s'est chargé peu à peu, à partir de Burckhardt, d'une fonction prophétique. Le présent article vise à revoir dans ce contexte les différentes idées reçues sur le texte mirandolien. Il est ainsi permis de réévaluer chez Pic, entre autres, le retour au latin classique, l'ontologisme, l'orthodoxie religieuse et l'univers cabalistique. Rien dans une telle relecture ne confirme la légende mirandolienne.

Dersonnage de légende peu à peu retourné à l'oubli, Jean Pic de la Mirandole a été "ressuscité" par les soins de Jacob Burckhardt qui, avec Jules Michelet, dit-on, "inventa" la Renaissance. ${ }^{2}$ C'est bien à la figure de Jean Pic, en effet, qu'en sa magistrale étude de La civilisation de la Renaissance en Italie (1859), l'historien suisse fera appel pour symboliser la spécificité et la riche diversité de l'humanisme du Quattrocento - mais, de toute l'oeuvre du Mirandolien, il ne retiendra que la seule Oratio de hominis dignitate, et de ce discours aux multiples facettes, il ne citera que les quelques paroles, désormais célèbres, par lesquelles Dieu, s'adressant à Adam, lui confère le privilège unique de la liberté:

Je t'ai placé au milieu du monde afin que tu puisses plus facilement promener tes regards autour de toi et mieux voir ce qu'il renferme. En faisant de toi un être qui n'est ni céleste ni terrestre, ni mortel ni immortel,j'ai voulu te donner le pouvoir de te former et de te vaincre toi-même; tu peux descendre jusqu'au niveau de la bête et tu peux t'élever jusqu'à devenir un être divin. En venant au monde, les animaux ont reçu tout ce qu'il leur faut, et les esprits d'un ordre supérieur sont dès le principe, ou du moins bientôt 
après leur formation, ce qu'ils doivent être et rester dans l'éternité. Toi seul tu peux grandir et te développer comme tu le veux, tu as en toi les germes de la vie sous toutes les formes. ${ }^{3}$

L'extrait, donné ici en entier, est bref et d'autant plus significatif que, dans son oeuvre, Burckhardt a rarement recours aux citations textuelles. À quoi vient s'ajouter que c'est l'ensemble de l'humanisme florentin que l'historien prétend évoquer à travers ces quelques lignes, dont la valeur emblématique ressort d'autant plus qu'en arrière-plan, le personnage historique, concret, de Jean Pic est laissé dans l'ombre: c'est à peine si Burckhardt le situe dans son temps, et il n'en décrit ni la vie, ni les autres oeuvres.

Et c'est ainsi que le Discours de la dignité de l'homme s'est peu à peu chargé d'un sens prophétique. Interprété selon les paradigmes qui ont donné corps à notre vision de la Renaissance, sa lecture ne pouvait que contribuer à consolider ces mêmes paradigmes, en une rétroaction sans fin. Quant à Jean Pic lui-même, on ne voit le plus souvent en lui que l'archétype imaginaire de l'humanisme du Quattrocento: il sera pour toujours ce héros prométhéen, précurseur prophétique de nos modernes émancipations et la simple évocation de son Discours suffira à faire surgir cette ambiance particulière, faite de recherche, d'inquiétude, de dynamisme, de rejet du passé et de retour aux âges classiques, d'anticipation, de crainte, d'angoisse et d'attentes inouïes, associée à ce renouveau dont Florence fut le berceau. ${ }^{4}$

Or, qu'en est-il du personnage réel de Jean Pic de la Mirandole? Dans quelle mesure est-il légitime de voir en lui le porte-parole exemplaire de cet humanisme italien, dont, au dire de nul autre que Garin, l'Oratio de hominis dignitate aurait été l'éclatant manifeste? C'est ce que l'on se propose d'évaluer ici et, pour ce faire, nous pourrons nous limiter à ne considérer, essentiellement, que le seul Discours, pour l'évidente raison que le mythe mirandolien s'est formé, exclusivement, à partir de ce texte. Jamais, en effet, dans cette optique, n'est-il référé à quelque autre écrit de Jean Pic: les Disputationes, le Commento, l'Heptaplus, les Conclusiones, les autres oeuvres du Mirandolien sont toujours laissées pour compte. Et, en effet, seul le Discours, et non tout le Discours, mais seul son premier tiers, et de ce premier tiers, seul le bref passage cité, peut donner lieu à une lecture "mythifiante," à condition encore que cette lecture soit suffisamment orientée par ce que l'on désire y découvrir. Rappelons d'emblée, pour remettre l'Oratio dans son contexte, qu'elle ne fut jamais prononcée par Pic, ni publiée de son vivant, le débat auquel le Discours devait servir de préambule ayant été interdit - et ceci, déjà, souligne l'aspect artificiel et "construit" de ce qu'il faut bien appeler le "mythe mirandolien." 
Par ailleurs, la simple analyse du Discours, si minutieuse soit-elle, ne permettra d'en dévoiler la charge mythique que dans la mesure où ses résultats auront été confrontés à ce qu'évoque, en général, la notion d' humanisme ellemême. Plutôt que de tenter une définition précise de ce vaste mouvement socio-culturel, on préférera s'en tenir ici, à titre indicatif, à cette conception de l'humanisme qu'expose Nicola Abbagnano dans le long article qu'il avait rédigé à la demande de la très respectée Encyclopedia of Philosophy ${ }^{5} \mathrm{La}$ référence générale à cet article nous semble d'autant plus justifiée que Nicola Abbagnano, lui-même philosophe italien d'envergure, connaît fort bien l'histoire de la philosophie et de la culture en général — tout en n'étant pas, au sens strict, ou étroit, du terme, un spécialiste de la Renaissance, encore moins de Jean Pic de la Mirandole. On peut donc s'attendre à ce que son article traduise de façon intelligente ce que le terme humanisme évoque au sein de la culture "savante," mais non spécialisée, où survit principalement le nom de Jean Pic sous son affabulation mythique. D'autre part, exemple en lequel on verra un échantillon typique de cette résurgence mythique, lorsque dans le cours de ce même article, Abbagnano veut illustrer de façon particulièrement suggestive ce qu'il entend par "renouveau humaniste," il s'en remet, lui aussi, à cette même et unique citation, à laquelle Burckhardt avait eu recours. A son insu donc, Abbagnano témoigne encore de la puissance évocatrice du mythe né de l'Oratio.

La démarche que nous suivrons ici est fort simple. Nous relèverons, d'une part, quelques-uns des traits les plus caractéristiques, ou des idées les plus habituellement reçues concernant l'humanisme, telles qu'on pourrait les inventorier chez Abbagnano, pris à titre d'exemple, et nous les comparerons ensuite à ce que l'on peut lire dans l'Oratio, de tous les écrits de Pic celui, donc, qui se prête le mieux à l'interprétation mythifiante, en fait, le seul qui s'y prête tant soit peu. L'inventaire complet de ces idées reçues serait très vaste; on s'en tiendra à la discussion de certaines d'entre elles seulement, qui paraissent les plus significatives.

\section{L'Oratio de hominis dignitate, fondement du mythe mirandolien}

\section{Retour au latin classique}

Le caractère le plus immédiatement évident de la littérature humaniste est la volonté affichée de remonter, par delà le latin "décadent" de la scolastique, aux sources les plus authentiques de l'héritage classique. Chez certains, tel Ermolao Barbaro, par exemple, cette obsession du retour aux sources classiques procédait du seul souci littéraire de retrouver une élégance formelle, seule 
garante, affirmait-il, d'immortalité littéraire. ${ }^{6}$ Les esprits plus réfléchis, cependant, $y$ voyaient la condition sine qua non de renouer, après l'interruption médiévale, avec l'idéal de l'ancienne paideia grecque. Celle-ci présupposait, croyait-on, que, seuls, les arts libéraux permettent à l'homme de transcender sa nature animale, que seule la maîtrise de ces arts le rend véritablement et pleinement homme, établissant ainsi entre l'homme et l'animal une différence, dirions-nous, de culture plutôt que de nature, - ce qui est, en effet, très “moderne." D'où le privilège accordé aux "humanités," "fondé, comme le dit Abbagnano, sur la conviction que seules ces disciplines donnent à l'homme l'éducation véritable qui lui permettront d'être pleinement libre."

Or, à en juger par son élégance littéraire, le style de Jean Pic se conforme aux normes humanistes les plus exigeantes. L'Oratio, en effet, est sans conteste un des plus beaux textes que nous ait légués la littérature néo-latine. Pic y fait preuve de sa parfaite maîtrise de toutes les subtilités et nuances du latin le plus classique et son discours est un chef-d'oeuvre de haute rhétorique: ne peut-on voir là une garantie suffisante de l' "humanisme" de Pic?

Seulement, il y a dans cette rhétorique même, de la part de Jean Pic, comme une mise en garde qui nous incite à ne pas donner trop d'importance à l'Oratio, en tout cas, à ne pas la prendre à la lettre et, surtout, à ne pas y voir un traité d' anthropologie métaphysique, comme d'aucuns le voudraient. On se souviendra, en effet, qu'à peine un an plus tôt, soit en juin 1485, répondant à Ermolao Barbaro, Pic s'était élevé avec force contre l'usage, en philosophie, des techniques propre à la rhétorique. Le philosophe, en effet, doit convaincre par la seule force de son argumentation, jamais par les blanditiae de la forme. Et c'est bien selon cette maxime que procède le Mirandolien, qui, en tous ses écrits proprement philosophiques, fait appel à ce "style de Paris" tant honni par les humanistes, mais dont lui-même vantait la précision et la rigueur, seules qualités, qui, au plan de l'expression, sont exigées du philosophe. ${ }^{7}$

Quant à dire que seuls les arts libéraux permettraient à l'homme d'affirmer sa supériorité sur l'animal, voilà ce que Pic récuse expressément. Jouant sur la distinction entre homo, l'homme en tant que tel, et humanus, l'homme de culture, le lettré, Pic affirme que si les belles lettres caractérisent sans doute ce dernier, la philosophie, elle, est le propre de tout être humain en tant que tel. ${ }^{8}$ Elle est donc antérieure à toute culture, bien plutôt que d'en être un "épiphénomène," à quoi la conception humaniste, si on l'analyse, tendrait à la réduire. 


\section{Ontologisme et liberté}

Il est souvent affirmé, d'ailleurs à fort juste titre, qu'une des caractéristiques les plus nettes par lesquelles la "modernité" s'oppose, non seulement au moyen âge, mais à l'ensemble des âges antérieurs, est l'abandon de toute "perspective ontologique" - entendant par "perspective ontologique" que l'ordre des phénomènes, des apparences ou des existences concrètes serait prédéterminé par un ordre plus fondamental, l'ordre de l'être ou l'ordre des essences. Et on suppose alors que, Pic étant à l'origine de la modernité ou ayant eu, à tout le moins, l'intuition de ce qu'elle serait, il a dû reléguer aux oubliettes moyenâgeuses toute trace d'ontologisme.

Notons d'abord que, de tous les écrits de Pic, l'Oratio est certainement celui où se manifeste le plus clairement l'influence du néoplatonisme le plus authentique, celui de Plotin. Si Pic avait voulu critiquer l'ontologisme, il n'aurait pas pu plus mal choisir son cadre de référence, car la doctrine de Plotin est, de toutes les grandes conceptions classiques, celle qui, en chacun de ses développements, est le plus indissociablement reliée à la perspective ontologique. Une lecture même rapide et superficielle de l'Oratio confirmera que le cadre général au centre duquel Pic situe l'être humain, est sans doute possible un cadre ontologiquement ordonné. Pic précise, par exemple, que "ce qui fait la plante, ce n'est pas sa texture fibreuse, c'est sa nature engourdie et privée de sensibilité; la bête de somme, ce n'est pas le pelage, mais l'âme brute et sensuelle ..."9 C'est donc affirmer qu'il est un principe, autre que "la texture fibreuse" ou "le pelage" — c'est-à-dire autre que la matière qui le reçoit — qui détermine chaque être à être ce qu'il est, et donc aussi, par voie de conséquence, à agir selon ce qu'il est. Comment illustrer plus clairement que "l'essence précède l'existence," ce qui, indépendamment du vocabulaire utilisé, correspondait, de Platon à Plotin, à la conception largement prédominante de la philosophie grecque?

Il suit de l'antériorité ontologique de la "forme" sur la "matière" que le lieu précis comme la fonction propre qui, au sein de la totalité, incombe à chaque être particulier, seront également assignés et définis par l'essence particulière de cet être: l'essence n'est pas seulement descriptive, elle est prescriptive, selon l'adage agere sequitur esse. Et par conséquent, l'ordre du monde sensible sera, non pas cette ordonnance $a$ posteriori, née graduellement de la convergence des hasards ou de la rencontre aléatoire des atomes, que déjà imaginait Démocrite, il sera, tout au contraire, le reflet dérivé d'un ordre existant en soi; et, dès lors, la hiérarchie des êtres se traduira toujours en 
hiérarchie des valeurs, comme le symbolise excellemment l'image biblique de l'Échelle de Jacob qui, reprise par Pic, devient la métaphore centrale de l'Oratio.

Mais, dira-t-on, Pic était homme de son temps, et quel qu'ait été son génie, force lui était de s'exprimer dans le cadre philosophique de ce temps, en respectant les contraintes "ontologiques" que ce cadre lui imposait, surtout au sein de l'académie florentine, dominée par la forte personnalité de Marsile Ficin. Cette objection ne paraît pas recevable. Pic, en effet, a toujours fait preuve de la plus grande indépendance d'esprit à l'égard des différentes écoles de pensée qu'il avait, pendant près de dix années, étudiées de fort près. Or, dans l'éventail qui s'offrait à lui, il aurait pu choisir d'inscrire son discours sur la dignité de l'homme dans le cadre "moderne" du scotisme et du nominalisme, qui, à l'époque, prédominaient largement en théologie, particulièrement à la Sorbonne, ${ }^{10}$ où Pic venait d'achever une année d'étude. Or, cette via moderna, par le fidéisme qu'elle impliquait, allait conduire au rejet de toute référence ontologique, et les travaux de Lagarde, ${ }^{11}$ comme l' ouvrage plus récent de Louis Dupré, ${ }^{12}$ ont montré l'influence "laïcisante" que, dès le seizième siècle, cette orientation exerça sur l'ensemble de la vie sociale et politique. Or, si, plus tard, Pic semblera se rapprocher de cette via moderna, en 1486, il s'y opposait radicalement, comme il serait aisé de le montrer à partir de son Apologie. C'est donc à bon escient et en pleine connaissance de cause, peut-on croire, que Pic a choisi d'écrire son Oratio sur l'arrière-plan du néoplatonisme, arrière-plan, donc, profondément et radicalement ontologique.

Cependant, dira-t-on encore, ce que Pic veut souligner, c'est que l'homme, quoique situé en un cadre ontologique, fait exception à la règle générale. Il n'est, lui, "limité par aucune borne," nullis angustiis coercitus. Et c'est pourquoi il est libre et, en dignité, supérieur aux anges eux-mêmes.

Est-ce dire que cette liberté soit absolue, qu'elle soit une liberté créatrice, au sens moderne du terme? Gardons-nous de faire ce saut! "En l'homme qui naît, dit l'Oratio, le Père a déposé tous les types de semences et les germes de toutes les sortes de vie"13 C'est pourquoi l'homme, image du Macrocosme, peut être dit microcosme. Et sa liberté naît, non pas de l'absence de nature, mais au contraire, de la présence en lui des potentialités de toutes les natures. Aucune, donc, ne le prédétermine absolument et c'est pourquoi l'homme pourra librement faire choix de développer telle ou telle de ces potentialités. Mais l'éventail des choix possibles est donné, il n'est pas, de soi, illimité: première restriction qui s'impose à la liberté de l'homme, elle n'est pas liberté créatice. 
À quoi on ajoutera que, face à cette échelle des êtres qui est en même temps échelle de valeurs, l'homme ne conserve que la seule alternative d'accepter ou de refuser cette échelle: il peut, selon son choix, s'y élever ou s'y abaisser, mais il ne peut en aucun cas ni d'aucune façon la modifier, ni y substituer son ordre propre. . . En d'autres mots, le choix qui est proposé à l'homme est un choix d'ordre moral, un choix éthique; ce n'est aucunement un choix d'ordre ontologique ou métaphysique.

On sera alors forcé de reconnaître que, ramenée ainsi à de plus justes proportions, cette conception de la liberté ne diffère plus guère de celle que proposait déjà le cosmocentrisme hellénique. En effet, comme le disait très précisément René Schaerer, en un livre justement intitulé L'Homme devant ses choix dans la tradition grecque: "il y a à la limite deux situations opposées, dont les âmes se rapprochent plus ou moins: l'état d'affranchissement et l'état de servitude. Entre les deux, s'étend la zone des choix." ${ }^{14}$ Et c'est précisément en cette "latitude," en cette souplesse, que consistait, pour Plotin également, la liberté de l'âme lorsqu'elle tend vers son achèvement. L'homme, affirmet-il, "dont la place est entre les dieux et les bêtes" [Enn. III, 2, c.8], peut soit s'élever par l'ascèse vers les rangs supérieurs ou, au contraire, s'abandonnant aux pulsions de sa sensibilité, s'abaisser aux rangs inférieurs: que trouve-t-on de plus dans le discours que propose Jean Pic?

\section{Laïcisation ou orthodoxie?}

En fait, on trouve, en plus, dans ce discours, une restriction essentielle et fondamentale, que Plotin n'aurait pas admise. Le grand attrait que la doctrine plotinienne a toujours exercé sur les âmes mystiques est cette promesse de rendre proche et accessible à l'homme un Dieu pourtant ineffable. C'est bien ce que propose, par exemple, l'exhortation que l'on peut lire dans la cinquième Ennéade: "recherche Dieu avec assurance à l'aide d'un tel principe et remonte jusqu'à lui; il n'est pas du tout loin et tu y parviendras: les intermédiaires ne sont pas nombreux." ${ }^{15} \mathrm{C}$ 'est l'écho de cette assurance, "mystique" et non du tout "laïque," qui résonne dans tant de passages de l'Oratio et, en particulier, dans celui-ci:

Qui, laissant derrière lui tout ce qui est humain, méprisant les biens de la fortune, indifférent à ceux du corps, ne voudrait, dès ici-bas, devenir convive des dieux, et, gorgé du nectar de l'éternité, recevoir le don de l'immortalité, tout animal mortel qu'il soit? 
Et ce qui, précisément, enthousiasme Pic à la lecture des Ennéades, c'est l'assurance, tant de fois proclamée par Plotin, que la "conversion" de l'âme, le retour à Dieu, c'est-à-dire l'extase mystique, serait possible "dès ici-bas"adhuc degens in terris -, comme le souligne Pic dans le passage que l'on vient de lire. Cependant, malgré ses propensions naturelles et ses enthousiasmes encore intacts - Pic n'a éprouvé jusqu'alors aucune des désillusions que lui réserve la vie - il est un point en lequel il se dissocie de l'Alexandrin.

En effet, malgré les apparences, la doctrine de Plotin reste résolument positive. Pour lui, le désir de s'élever vers le Bien absolu n'implique, de soi, aucun dédain à l'égard du monde et de ses valeurs. Même s'il "semblait avoir honte d'être dans un corps," comme le disait Porphyre dès la première ligne de la biographie qu'il consacre à son maître. ${ }^{17}$ Plotin n'a jamais renoncé à cet optimisme ontologique radical, propre aux Grecs: tout être, même le moindre, est bon de par sa nature.

Pic, quant à lui, ne peut partager sans restriction cette vision optimiste: il ne manque pas de signaler que le monde dont il parle est un monde déchu, "tout entier au pouvoir du Malin." Doit-on y voir une influence de la gnose? Peutêtre. Toujours est-il que, sur cette question, le Mirandolien partage l'ambiguïté propre au Moyen Age, toujours écartelé sur le plan moral entre l'absolutisation manichéenne du contemptus mundi et la proclamation doctrinale de la bonté intrinsèque de l'oeuvre de Dieu. Si elle n'a pu être radicalement corrompue par la révolte de Lucifer et par le péché d'Adam, la création n'en a pas moins été profondément pervertie, et la tentation de "fuir le monde" semble avoir exercé sur Pic - et ce, dès l'Oratio, notons-le - une emprise aussi forte que celle de vouloir, "dès ici-bas," rejoindre la Jérusalem céleste. C'est l'écho de cette ambivalence que l'on peut percevoir jusque dans les paroles exaltées en lesquelles Pic décrit le dévoilement extatique auquel conduit la progression mystique:

Qui ne voudrait être atteint de cette folie socratique chantée par Platon dans le Phèdre, de façon à fuir en toute hâte, par tous les moyens à sa disposition, ce monde d'ici-bas qui est au pouvoir du Malin, et à s'élancer de la course la plus prompte vers la Jérusalem céleste? ${ }^{18}$

Une chose est claire: qu'ils soient d'inspiration gnostique, ou plus proches de l'orthodoxie, de tels passages - faut-il le dire? - seraient bien difficile à interpréter en un sens prométhéen. . ${ }^{19}$

D'ailleurs, Pic insère, dans le texte même de l'Oratio, une allusion évidente à la doctrine du péché originel. Lisons en leur version originale deux 
lignes essentielles de la citation donnée par Burckhardt: "Poteris in inferiora quae sunt bruta degenerare; poteris in superiora quae sunt divina ex tui animi sententia regenerari" ${ }^{20} \mathrm{Pic}$, ici, joue sur l'opposition grammaticale entre la voix passive - "poteris (...) regenerari," "tu pourras (. . .) être régénérê" — et la voix active - "poteris degenerare," "tu pourras dégénérer." ${ }^{21}$ En d'autres mots, de par ses propres forces, l'homme pourra sans doute s'abaisser jusqu'aux êtres inférieurs, mais pour accéder au rang de la divinité, il lui faudra l'aide d'une grâce essentielle. Celle-ci, cependant, nécessite le concours de l' homme et ne saurait être efficace que si l'homme, librement - "selon les voeux de son coeur" - l'attend et l'accueille: l'homme doit vouloir se laisser régénérer.

On retrouve ainsi les éléments-clés de la plus stricte orthodoxie chrétienne telle qu'elle était admise avant les controverses de la Réforme. Pour tout auditeur de Pic, le sens de cette apostrophe ne laissait place à aucune ambiguiité, et ce, d'autant moins que, depuis Isidore, le terme regeneratus désignait le baptisé, et que le baptême était le sacrement de la régénération. Que, cependant, l'on ne tienne pas compte de ce regenerari, et qu'on lui substitue regenerare, cette simple phrase, banale et orthodoxe sous son vernis oratoire, deviendra la proclamation d'un pélagianisme prométhéen: ${ }^{22}$ or, il faut bien constater que c'est précisément ce que font la majorité des traducteurs.

Soulignons que ce jeu des voix active et passive est une constante chez Pic; on le retrouve, par exemple, dans son Commentaire du Psaume $X^{23}$, dans ses Regulae $^{24}$ et, surtout, dans l'Heptaplus ${ }^{25}$ où il se double d'une véritable théologie de la grâce. On y lit, en effet, que le macrocosme se trouvant parfaitement représenté par le microcosme qu'est l'homme, la chute d'Adam a entraîné celle de l'univers en sa totalité. En contrepartie, sa rédemption par le Christ s'étend également à l'ensemble de la création. ${ }^{26}$

\section{Perennité du mythe}

On est donc obligé de conclure que, au vu des textes, seule une lecture superficielle et orientée de l'Oratio permet de voir en Jean Pic le héraut prométhéen des temps modernes. Pourtant, comme on l'a dit plus haut, ce mythe a une extraordinaire puissance d'auto-régénérescence et, malgré toutes les critiques qui, depuis une quarantaine d'années, en ont été faites, il reste toujours aussi vivace. Il est devenu une sorte d'entité anhistorique qui, planant au-dessus des temps, résiste et résistera sans doute indéfiniment à toute tentative de "déconstruction." En exemple de quoi, on peut citer la conférence donnée par August Buck lors de la séance d'ouverture du récent congrès de 
Mirandole. Se fondant, ici encore, sur le seul et même passage de l'Oratio, cité in extenso, avant de reprendre les clichés les plus habituels concernant Jean Pic, l'orateur alla jusqu'à prétendre que Pic aurait proposé "une déification de l'homme qui ne tient pas compte de la tache du péché originel, ni de la nécessité de la grâce divine, une rédemption opérée par l'homme et donc fondée sur la confiance humaniste en la perfectibilité de la nature humaine, que l'homme peut réaliser par ses forces propres." 27

Notons cependant que, nonobstant la lecture "prométhéenne" de l'Oratio qu'il vient de donner, August Buck souligne, quelques paragraphes plus loin, que Pic ne cessera, face à la commission d'enquête qui le harcèlera de ses critiques, "d'affirmer solennellement sa propre orthodoxie, dont il était totalement convaincu." Et le conférencier d'ajouter: "Les recherches modernes ne soulèvent aucun doute quant à son orthodoxie chrétienne" (Ibid.). ${ }^{28}$

Quelles qu'en soient les modalités, prenons acte de ce témoignage irrécusable de l'orthodoxie de Jean Pic. D'ailleurs, depuis de Lubac, ${ }^{29}$ celle-ci n'est plus guère remise en question. Certains, cependant, vont beaucoup plus loin en cette voie: non contents d'affirmer ou, éventuellement, de "démontrer" l'orthodoxie mirandolienne, ils font de Jean Pic le parangon de l'orthodoxie chrétienne elle-même et, dès lors, ils n'hésitent pas à voir en sa pensée, en son "système," la norme même qui permettrait, par comparaison, d'évaluer l'orthodoxie d'autres penseurs ou théologiens chrétiens.

Auteur d'une solide étude de la pensée théologique de Jean Pic, ${ }^{30} \mathrm{H}$. Reinhardt vient de publier un article au titre prometteur: "De illis Pici vestigiis quae in regno theologiae ac praesertim in provincia theologorum huius saeculi vicesimi supersunt."31 Après avoir exposé l'essentiel de la doctrine mirandolienne et insisté sur 1" "onto-théologie" christocentrique de cette doctrine, qu'il estime parfaitement conforme aux plus purs principes pauliniens, Reinhardt passe successivement au crible d'une sévère critique les pensées ou systèmes de Teilhard de Chardin, de Hans Urs von Balthasar et de Karl Rahner. Dans chaque cas, s'il fait état de certaines similitudes apparentes entre les thèses de ces auteurs et la doctrine de Jean Pic, c'est pour mieux souligner que de telles ressemblances sont superficielles ou contingentes, ou qu'elles résultent simplement d'un fond théologique commun. Ce qui conduit notre auteur à cette première conclusion: “. . . il me faut donc déclarer que les dissemblances entre ces auteurs contemporains et le théologien que fut Pic l'emportent nettement sur leurs ressemblances." 32 En suite de quoi, chacune des "ressemblances" signalées est portée au crédit de ces théologiens contemporains, alors que toute différence leur est imputée négativement. En d'autres mots, 
toujours selon Reinhardt, ce qu'il y a de juste chez ces auteurs se trouvait déjà chez Pic, tandis que ce en quoi ils dévient du modèle mirandolien est apporté en témoignage de la faiblesse de leur théologie. . . Et l'auteur de terminer par cette conclusion globale: "[Pic] est le miroir et l'exemple même d'une

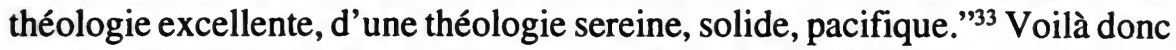
une autre facette, combien inattendue, du mythe mirandolien!

\section{Eschatologie ou histoire et politique?}

Dans l'ontologisme grec, les espèces sont, comme le monde, éternelles et donc immuables. Et cela vaut également pour l'espèce humaine, ce qui exclut non seulement toute révolution, mais également toute évolution et même toute histoire. Certes, dans la conception chrétienne, l'histoire apparaît, mais c'est une "histoire sainte," dont les temps forts sont la création, la chute, la rédemption et, ultimement, la parousie. Il s'agit donc d'une histoire des rapports entre l'humanité et Dieu, non d'une histoire autonome de l'humanité, d'une histoire profane. Et c'est pourquoi, quelle que soit la liberté dont l'homme ait reçu le privilège, ce sera toujours une liberté normée, on l'a vu, et une liberté individuelle. En d'autres mots, ces potentialités qui délimitent le champ de la liberté humaine et qui sont inhérentes à "tout homme qui naît," ces potentialités sont, elles, immuables. Telle est la condition humaine, selon Pic, et il n'est au pouvoir d'aucun homme, pas plus qu'il n'est au pouvoir de l'humanité dans son ensemble, de la modifier. Ce qui limite, évidemment, le sens du politique.

Dans un ordre à fondement ontologique, en effet, tout segment de la réalité participe de la totalité, y trouve sa place et sa fonction prescrites, et en reflète la structure. Dans ce cadre, pour paraphraser Abbagnano, les institutions humaines fondamentales - société, Église, État - se font les expressions locales et les garantes d'une hiérarchie cosmique, qui s'impose à l'homme et qu'il lui serait donc vain de vouloir modifier. Dans cette perspective, l'action politique ne pourra avoir d'autre finalité propre que d'instaurer, de conserver ou de restaurer une organisation sociale dont les normes se trouvent inscrites dans la nature. Plus généralement, toute action trouvera sa norme dans la vision d'une essence préexistante qui la transcende et qu'il importera d'abord de déchiffrer, et le projet humain se limitera dès lors à incarner cette essence idéale.

On comprend donc que, dans une telle conception, l'éthique se fonde sur une métaphysique et que, parallèlement, ou par voie de conséquence, la primauté soit accordée à la contemplation plutôt qu'à l'action, à la vie 
contemplative plutôt qu'à la vie active, à l'otium plutôt qu'au negotium. À l'inverse, raisonne-t-on, l'abandon de la perspective ontologique devrait se traduire par le renversement de cet ordre des valeurs et des priorités qu'il implique. C'est ce à quoi se seraient employés les humanistes, ouvrant ainsi la voie à la "modernité."34

Qu'en est-il alors de Jean Pic? Trouvera-t-on chez lui cette subordination de la vie contemplative aux exigences de la vie active, au profit, en particulier, de la vie politique, subordination qui impliquerait, fondamentalement, la dévaluation de la métaphysique au profit de l'éthique?

La réponse de l'Oratio ne laisse place à aucune ambiguiité. Se référant à la succession des ordres angéliques, dont les montées et les descentes alternent tout au long de l'Échelle de Jacob, Pic reprend la symbolique et la hiérarchisation traditionnelles, qui est celle du Pseudo-Denys:

Dédaignons les choses de la terre, méprisons celles du ciel et, laissant derrière nous tout ce qui est du monde, prenons notre envol vers le sénat d'au-delà des mondes, tout proche de la très éminente divinité. Là, comme les mystères sacrés nous la transmettent, les Séraphins, les Chérubins et les Trônes occupent les premières places; ignorant ce que c'est que de céder et ne supportant pas d'être aux deuxièmes places, cherchons à égaler la gloire et la dignité de ceux-ci. Quand nous l'aurons voulu, nous ne leur serons inférieurs en rien [. . .] Si donc, engagés dans la vie active, nous montrons pour les réalités de ce bas-monde un souci proportionnel à leur juste valeur, nous serons affermis par l'inébranlable solidité des Trônes. Si, libérés de l'action, méditant dans l'oeuvre l'artiste, dans l'artiste l'oeuvre, nous avons comme souci le repos de la contemplation, nous rayonnerons par toute notre personne la lumière des Chérubins. Si nous brûlons d'amour pour l'artiste lui-même exclusivement, le feu dévorant que celui-ci excite va tout à coup nous embraser, à l'image des Séraphins. ${ }^{35}$

L'extase, la contemplation, l'action, telles sont donc, en ordre décroissant, les valeurs que propose Pic dans son Oratio.

Et ce sont les mêmes stades que Pic énumère lorsqu'il décrit les étapes successives de la démarche qui conduira l'initié à l'ultime révélation. ${ }^{36} \mathrm{Ce}$ n'est que purifiée par la science des moeurs et de la logique que l'âme, "assagie et apaisée," pourra s'ouvrir à la lumière de la "philosophie naturelle," qui la conduira enfin, par la connaissance des choses divines, à la félicité théologique.

Logique et éthique, chacune en leur domaine propre, sont les préambules à la connaisance philosophique qui, elle-même, subordonne la philosophie de la nature à la "philosophie première," c'est-à-dire, la théologie ou la 
métaphysique: voilà, jusqu'ici, une démarche tout aristotélicienne, qui place la béatitude dans la pure contemplation. S'il est vrai, comme le prétend encore Abbagnano, que les humanistes opposaient l'Aristote éthicien des temps modernes au physicien et au métaphysicien de la tradition médiévale, on voit déjà de quel côté il faudra situer Pic.

Mais l'Oratio, plus qu'aristotélicienne, est néoplatonicienne, et Pic, fidèle à Plotin, y propose une démarche plus spiritualisante que celle d'Aristote. Pour ce dernier, en effet, la contemplation est l'aboutissement ultime de la philosophie et de toute sagesse humaine. Pour Plotin, par contre, et donc pour Pic, elle n'est que l'instrument qui permettra à l'âme, purifiée et enfin détachée de tout lien terrestre, de s'unir à Dieu dans l'extase mystique.

Tel est donc l'ordre théorique qui se dégage d'une lecture tant soit peu attentive de l'Oratio, mais tel également est l'ordre de valeurs que Pic s'enorgueillit d'avoir constamment respecté dans sa vie pratique. En effet, s'insurgeant contre tout pragmatisme en philosophie, Pic se fait gloire de n'avoir jamais "philosophé que pour philosopher":

jamais, dit-il, je n'ai espéré ou voulu de mes études ou de mes travaux d'autre récompense ou d'autre fruit que la culture de l'esprit et que cette connaissance d'une vérité qui fut toujours le principal objet de mon désir. J'en ai toujours été avide et amoureux fou, de sorte que, laissant de côté toute préoccupation d'ordre public ou privé,je me suis livré tout entier au repos de la contemplation, contemplandi otio totum me tradiderim. ${ }^{37}$

Nous retrouvons-là cette même attitude de fier détachement que Pic avait déjà proclamée dans sa lettre à Andrea Corneo, écrite en ce même automne 1486 alors qu'il rédigeait l'Oratio.

Corneo avait vivement incité Pic à abandonner enfin ces stériles études philosophiques, pour mettre ses talents au service de quelque prince: ainsi, au moins, Pic ferait-il oeuvre utile. C'est profondément vexé, touché en ses plus intimes convictions, que Pic réplique à son ami:

Tu écris qu'il est temps que je me mette au service de l'un des princes régnant en Italie. C'est que tu ne connais pas l'opinion que les philosophes ont d'euxmêmes, eux qui, comme Horace, s'estiment rois parmi les rois. Ils ne peuvent se soumettre aux coutumes serviles; ne quittant guère leurs cénacles, se satisfaisant de la paix de leur esprit, ils se suffisent à eux-mêmes et ne cherchent rien hors d'eux-mêmes; [...] Fidèle à cette vision des choses, j' ai toujours, aux cours princières, aux charges publiques, au désir de plaire et aux faveurs des curies, préféré ma cellule et mes études, les délices de mes livres et la paix de mon âme. ${ }^{38}$ 
On comprend, dès lors, qu'après l'échec radical de la dispute romaine et les déboires, condamnation et exil qu'il lui valut, Pic se soit de plus en plus retiré du monde, qu'il ait recherché la paix et que, pour ce faire, il ait partagé son temps entre sa thébaïde de Corbola et ses retraites fréquentes à l'abbaye de Fiesole, sur les hauteurs florentines. Mais voilà une attitude qui ne concorde guère avec l'“activisme" habituellement associé à l'éclosion des temps modernes. ${ }^{39}$

\section{La "chrématistique"}

Peu après la mort de sa mère, qui survint en août 1478 alors qu'il n'avait pas quinze ans, Pic hérita du tiers de la vaste fortune familiale, aussi ne connaîtrait-il jamais, même aux périodes les plus sombres de son existence, les difficultés matérielles de la vie. Sa perception de la réalité en restera profondément marquée, et sans doute peut-on y voir l'explication de ce dédain qu'il lui était impossible de ne pas ressentir à l'égard des dimensions plus terreà-terre de l'existence. ${ }^{40} \mathrm{C}$ 'est ce dédain qui le rendait aveugle, par exemple, aux promesses d'avenir que recelait le renouveau commercial et financier dont Florence, en cette seconde moitié du quinzième siècle, était le théâtre privilégié. C'est cet aveuglement qui explique le mépris tout "platonicien" en lequel Pic tenait les "mathématiques des marchands," qui, pourtant, étaient en plein développement dans les botteghe florentines, et dont allait naître notre algèbre. ${ }^{41}$ Présentant dans l'Oratio ses Conclusions de mathématiques, Pic rappelle que Platon lui-même avait écrit: "de tous les arts libéraux et de toutes les sciences spéculatives, la principale et la plus divine consiste à savoir nombrer." D'un même trait, cependant, Pic souligne que

de telles affirmations ne peuvent absolument pas être vraies, si l'on entend par art de nombrer, cet art en lequel les marchands d' aujourd' hui sont passés maîtres, comme l'atteste Platon, lorsqu'il nous avertit clairement de ne pas confondre la divine arithmétique dont il parle avec l'arithmétique des marchands. ${ }^{42}$

On le voit donc, l'ordre de valeurs du Mirandolien est parfaitement fidèle au cadre aristotélicien, qui mettait au premier rang la vie contemplative, l'otium, au second rang, la vie politique, le negotium, et, en dessous même de la vie militaire et de la quête du plaisir, la "chrématistique," cette recherche du profit qu'Aristote rejettait si dédaigneusement dans son Éthique à Nicomaque [I, 1096a].

$\mathrm{Si}$, comme le disait encore Abbagnano, les humanistes prétendaient, à l'encontre de la mentalité médiévale, revaloriser la fonction commerciale et 
légitimer le souci du bien-être matériel, il faudrait bien, à nouveau, exclure Pic de leurs rangs. . .

\section{Magie et Cabale}

L'intérêt soutenu que Pic manifeste à l'égard de la magie et de la Cabale figure sans aucun doute parmi les aspects les plus fascinants de son mythe. Pic, en effet, consacre plusieurs de ses Conclusions à l'un et à l'autre $\operatorname{art}^{43}$ et il les présente longuement dans l'Oratio. C'est en particulier la signification profonde de cet aspect de l'oeuvre mirandolienne que F. Yates voulut jadis mettre en lumière, proclamant en un essai célèbre:

The profound significance of Pico della Mirandola in the history of humanity can hardly be overestimated. He it was who first boldly formulated a new position for European man, man as Magus using both Magia and Cabala to act upon the world, to control his destiny by science. ${ }^{44}$

On peut certes établir une sorte de parallèle entre l'attitude technicienne de notre mentalité moderne, et l'attitude du magicien. Ne s'agit-il pas, dans un cas comme dans l'autre, d'appliquer une connaissance théorique des lois de la nature à la domination de cette dernière? D'où l'affirmation enthousiaste de Mme Yates, peut-être, - mais il faudra alors reconnaître que cette volonté du Mage est, comme la magie, de tous les temps et de tous les peuples, et non propre à Jean Pic. Par ailleurs, que celui-ci se soit laissé fasciner par les sciences occultes, au moins pendant la brève période qui va de 1485 à 1490 environ, voilà qui n'a rien d'étonnant, en cette fin du quinzième siècle, et particulièrement au sein des cercles florentins que Pic fréquentait assidûment: c'est l'attitude contraire qui eût été étonnante!

Guère originale non plus est la distinction sur laquelle insiste Pic, qui oppose la légitime magie "naturelle," à la goeteia, cette sorcellerie ou magie noire dont l'efficacité dépend tout entière de l'intervention du démon.

Ce qui mérite néanmoins de retenir l'attention, c'est la justification théorique qu'il tente de donner à la magie. En effet, il reprend à son compte cette même justification que Plotin expose longuement dans sa IVe Ennéade. ${ }^{45}$ C'est cette "théorie" que Pic paraphrase, tant dans l'Oratio que dans ses Conclusions magiques. On voit donc que son exaltation de la magie s'inscrit directement dans le cadre "orphique" de la cosmologie néoplatonicienne, qu'elle illustre et dont elle est une conséquence directe.

Sans doute Pic a-t-il pu espérer, dans son enthousiasme initial, que la magie lui fournirait comme une confirmation a posteriori de cette cosmologie 
néoplatonicienne et de la métaphysique qu'elle présuppose. Par tempérament, cependant, Pic était essentiellement contemplatif, et si les fondements théoriques que la magie recevait chez Plotin ont pu l'intéresser au plus haut point, il semble qu'il ait toujours laissé à d'autres le soin d'en tenter la vérification empirique. ${ }^{46}$ C'est, d'ailleurs, ce que, plus tard, confirmera Benivieni. ${ }^{47} \mathrm{De}$ même peut-on aujourd'hui être fasciné par l'efficacité de la pensée scientifique, tout en ne s'intéressant qu'à la vision théorique qu'elle suppose et sur laquelle elle se fonde.

\section{Conclusion}

Arrêtons ici ce survol critique des aspects les plus marquants du "mythe" mirandolien: on a pu constater que ce mythe est né d'une lecture "burckhardtienne" d'un bref passage de l'Oratio. S'il est lu dans sa totalité, cependant, et s'il est replacé dans son contexte historique, philosophique et théologique, rien, dans ce discours posthume, ne justifie cette vision d'un Pic prométhéen, et rien, même, ne permet de dire que Pic s'y soit montré "moderne," quelque acception que l'on donne à ce mot. ${ }^{48}$

Ajoutons en terminant que la condamnation, d'abord partielle puis globale, des Conclusiones a pu sembler confirmer l'image d'un Pic “faustien." Quoiqu'il y faudrait une analyse qui dépasserait largement le cadre du présent article, ${ }^{49}$ on peut montrer que tout au long de leur enquête, ce furent les juges de Jean Pic à défendre l'orientation qui, à l'époque, passait pour "moderne," alors que Pic, beaucoup plus proche de la tradition thomiste, se montrait, lui, conservateur. . . Voilà qui, une fois de plus, n'est guère conforme à son mythe.

\section{Université de Sherbrooke}

\section{Notes}

1. Ce texte, en version abrégée, a fait l'objet d'une communication lors de la session conjointe de la Société Canadienne d'Études de la Renaissance et de la Società Canadese per gli Studi di Italianistica, Calgary, 5 juin 1994. Les références au texte de Pic se font à l'édition Garin, Florence, 1942, pour l'Oratio de hominis dignitate; pour l'Apologia, à l'édition Vasoli des Opera Omnia, repr. anast., Hildesheim, 1969.

2. Cf. Lucien Febvre, "Comment Jules Michelet inventa la Renaissance," Studi in onore di Guido Luzzatto, III, Milan, 1950, pp. 1-14.

3. Jacob Burckhardt, La civilisation de la Renaissance en Italie, trad de H. Schmitt, revue et corrigée par R. Klein (Paris, Plon, 1958), pp. 286-287. 
4. Ainsi, exemple parmi bien d'autres, de Marguerite Yourcenar. Son Oeuvre au Noir, roman admirable par ailleurs, met en scène le Mage Zénon, héraut d'une nouvelle sagesse, faite de lucidité, de stoïcisme et de maîtrise de soi, pour laquelle d'ailleurs il sera mis à mort par les suppôts d'une tradition obscurantiste. Quoique ce Zénon n'ait guère en commun avec Jean Pic que d'être son contemporain approximatif et fictif, le drame qu'il va vivre est évoqué, en son essence, par les mêmes paroles de l'Oratio, qui, mises en exergue, parrainent son récit. Elles suffisent: tout y est dit.

Autre exemple, dans un domaine fort différent. Lorsque l'éminent théologien Edward Schillebeeckx veut représenter l'esprit moderne dans ce qu'il a de plus caractéristique, pour faire ressortir ce par quoi cet esprit se distingue et s'oppose le plus résolument à la mentalité médiévale, c'est à ces mêmes quelques lignes qu'il fera encore appel, car, prétend Schillebeeckx, Jean Pic y aurait "annoncé de manière prophétique un avenir proche où l'homme, grâce à la science et à la technique, prendrait son sort en main. (Edward Schillebeeckx, L'Histoire des hommes, récit de Dieu [Paris, Éd. du Cerf, 1992], p. 27. Notons que le dominicain situe le Discours "au seuil des Temps modernes, en 1442 pour être précis," alors qu'il date de décembre 1486).

5. (New York \& London, Macmillan \& The Free Press, 1972), vol. 4, pp. 69-72.

6. C'est ainsi qu'après avoir félicité Pic pour sa maîtrise du grec "sans laquelle il n'aurait été rien" - sine quibus nihil eras futurus -, Ermolao Barbaro précise qu'en tant de siècles, aucune oeuvre latine n'a excellé car aucun de leurs auteurs ne maîtrisait le grec — nullius latina lingua tot saeculis extare monumenta, qui litteris graecis caruerit -, pour terminer sa diatribe en affirmant que seul un style clair et élégant, au moins tel que le pratiquait les Pères grecs et latins, peut procurer à son auteur une gloire éternelle - sermo nitidus et elegans, saltem purus et castus, qualis vel in auctoribus christianis, Graecis Latinisque, perspicitur, laudem et memoriam sempiternam scriptoribus conciliat. Cf. Ermolao Barbaro: Epistolae, Orationes et Carmina, textes établis par V. Branca (Florence, Bibliopolis, 1943), Vol. I, pp. 85-87.

7. On connaît la mise en garde explicite que donne Pic au début de ses Conclusiones, auxquelles l'Oratio aurait dû servir de présentation littéraire: "En formulant [ces conclusions], ce n'est pas l'éclat de la langue romaine qu'il a reproduit, mais le mode d'expression des plus célèbres maîtres parisiens de la dispute, car c'est celui qu'utilisent à peu près tous les philosophes de notre époque" - "In quibus recitandis non Romanae linguae nitorem, sed celebratissimorum Parisiensium disputatorum dicendi genus est imitatus, propterea quod eo nostri temporis philosophi plerique omnes utuntur," Conclusiones sive theses DCCCC, texte établi par Bohdan Kieszkowski (Genève, Droz, 1973), p. 27.

8. "Non est humanus qui sit insolens politioris litteraturae; non est homo qui sit expers philosophiae," Epistola de genere dicendi philosophorum, dans Prosatori latini del Quattrocento, a cura di Eugenio Garin (Milan - Naples, Riccardo Ricciardi), p. 820.

9. "Neque enim plantam cortex, sed stupida et nihil sentiens natura; neque iumenta corium, sed bruta animalis et sensualis. ..," E. Garin, p. 108.

10. C'est ce que Pic lui-même affirme à plusieurs reprises dans son Apologie, ainsi: “[. . .] la voie courante, $i$. e. la voie de Scot, de Bonaventure, de tous les scotistes et de presque tous les nominaliste [...]. J'appelle 'voie courante des théologiens' celle qui est couramment admise 
aujourd' hui à Paris où règne principalement l'étude de la théologie" - "[...] communis via, id est via Scoti, Bonaventurae \& omnium Scotistarum \& quasi omnium Nominalium [. . . ] Communem viam theologorum appellans, quae communiter nunc tenetur Parisiis, ubi praecipue viget studium theologiae [. . .]" Apologia, Opera omnia, fol. 134-135.

Également: fol. 127, 138. . .

11. Georges de Lagarde, La naissance de l'esprit lä̈que au déclin du Moyen Âge (Louvain Paris, Nauwelaerts - Béatrice Nauwelaerts, 1956-1963).

12. Louis Dupré, Passage to Modernity: An Essay in the Hermeneutics of Nature and Culture (New Haven \& London, Yale University Press, 1993). Dupré attribue à la "théologie nominaliste du XIV" siècle" une influence déterminante dans ce "passage à la modernité."

13. "Nascenti homini omnifaria semina et omnigenae vitae germina indidit Pater," E. Garin, p. 106.

14. René Schaerer, $L$ 'homme devant ses choix dans la tradition grecque (Louvain, Presses de l'Université de Louvain, 1963), p. 101, souligné par moi.

15. Enn. V, 1, c. 3, 1. 2-4, trad. Bréhier (Paris, Les Belles Lettres).

16. "Quis humana omnia posthabens, fortunae contemnens bona, corporis negligens, deorum conviva adhuc degens in terris fieri non cupiat, et aeternitatis nectare madidus mortale animal immortalitatis munere donari?," E. Garin, p. 122.

17. Vie de Plotin, dans Ennéades, trad. Bréhier, vol. 1, p. 1.

18. "quis non Socraticis illis furoribus, a Platone in Phaedro decantatis, sic afflari non velit, ut alarum pedumque remigio hinc, idest ex mundo, qui est positus in maligno, propere aufugiens, ad caelestem Hierusalem concitatissimo cursu feratur?," E. Garin, 122.

19. C'est ce que note également Louis Dupré, Op. cit., p. 125.

20. E. Garin, p. 106.

21. Notons que la conjonction du rôle actif de la volonté (poteris... ex tua anima sententia) avec la passivité du regenerari rappelle très précisément cette "synergie mystérieuse de la grâce et du libre arbitre" en laquelle A. Solignac voit un aspect essentiel de la conception augustinienne: Oeuvres de Saint Augustin, Vol. 13, Les Confessions i-vii (Paris, Desclée de Brouwer), p. 33.

22. On peut exclure que ce passif soit dû à une modification ultérieure ou à quelque lapsus calami, car les deux versions de l'Oratio - soit sa version finale et sa première ébauche, retrouvée et publiée par Eugenio Garin - portent regenerari, cf. "La prima redazione dell'Oratio de hominis dignitate," dans La cultura filosofica del Rinascimento italiano (Florence, Sansoni, 1979), p. 234.

23. "Tum vero id maxime fuit theologia scientia ad maiora nos provehens et non solum ad id cohortans ut integram retineamus humanam dignitatem, ne vel ab homine degeneremus in brutum, sed ut sancta aemulatione divinarum mentium, quarumilla nobis naturam demonstrat ex terrenis hominibus in coelestes homines regeneremur," cf. Fernand Roulier, Jean Pic de la Mirandole (1463-1494), humaniste, philosophe et théologien (Genève, Slatkine, 1989), p. 446, n. 83 (souligné par moi). 
24. Pic n'y souligne plus guère le rôle du choix humain puisque, s'adressant à Dieu, il l'implore en ces termes: “. . . sed tu es, qui trahes me ad te, per gratiam tuam ..." (Garin, p. 266).

25. Hept. III, 7: “... sed et nos omnes, quibus data potestas filios Dei fieri per gratiam cuius dator est Christus, supra angelicam dignitatem evehi possumus," (Garin, p. 266). Notons qu'ici encore le traducteur italien rend le passif evehi possumus par l'actif possiamo elevarci.

26. Hept. VII, Prooem. (Garin, pp. 328-334, passim). La notion de "rédemption cosmique" trouve sa source dans l'optimisme onto-théologique de Bernard Sylvestre, cf. L. Dupré, $O p$. cit., p. 33.

27. "Una deificazione dell' uomo che non tiene conto della macchia del peccato originale e della necessità della grazia divina, une redenzione ad opera dell'uomo fondata dunque sulla fiducia umanistica nella perfettibilità della natura umana attuabile con le proprie forze" (photocopie du texte tel que lu, p. 11). - August Buck, "G. Pico della Mirandola e l'antropologia dell'umanesimo italiano,"Séance d'ouverture, Congrès international d'études sur Jean Pic de la Mirandole à l'occasion du cinquième centenaire de sa mort (1494-1994), Mirandola, 4-8 octobre 1994. A. Buck ayant été retenu par la maladie, sa conférence fut lue in absentia par le président de séance Enzio Raimondi.

28. "Pico si difese appassionatamente contro l'accusa di eresia nella "Apologia" nella quale inserì dei brani della "Oratio," affermandovi solennemente la propria ortodossia di cui lui era convintissimo. Anche le ricerche moderne non sollevano dubbi sulla sua ortodossia cristiana," A. Buck, Ibid., p. 13. Notons en passant que l'on voit mal comment l'auteur pourrait concilier le pélagianisme radical que sa première citation attribue à Pic avec l'orthodoxie chrétienne proclamée par la seconde. . .

29. Henri de Lubac, Pic de la Mirandole (Paris, Aubier Montaigne, 1974).

30. Heinrich Reinhardt, Freiheit zu Gott, Der Grundgedanke des Systematikers Giovanni Pico della Mirandola (1463-1494) (Weinheim, V.C.H., Acta Humaniora, 1989) - Notons ce sous-titre, particulièrement révélateur.

31. Cf. Vivens Homo, Rivista teologica fiorentina, 5/2 (1994), pp. 269-298.

32. "Finem facere nequeo, priusquam declaraverim dissimilitudinem horum auctorum recentiorum a Pico theologo multo vivaciorem esse quam similitudinem cum Pico," Heinrich Reinhardt, Art. cit., p. 295.

33. "Speculum enim est [Picus] et specimen theologiae optimae, theologiae quietae, solidae, pacificae," Ibid., p. 298.

34. Comme l'écrivait très justement Danièle Letocha: "la première tâche consiste donc à faire le vide en disqualifiant les normes, les modèles idéaux, les données ontologiques issus de la structure contemplative-passive ... La modernité se polarise donc autour de l'exigence d'un FAIRE qui se veut maîtresse de son origine, qui doit donc rendre compte de son propre fondement au degré zéro" - "Comment définir la modernité quand on est encore régi par ses impératifs?," Carrefour, vol. XIII/1 (1991), pp. 8-9.

35. "Dedignemur terrestria, caelestia contemnamus, et quicquid mundi est denique posthabentes, ultramondanam curiam eminentissimae divinitati proximam advolemus. Ibi, ut sacra 
tradunt mysteria, Saraphin, Cherubin et Throni primas possident; horum nos iam cedere nescii et secundarum impatientes et dignitatem et gloriam aemulemur. Erimus illi, cum voluerimus, nihilo inferiores [...] Ardet Saraph caritatis igne; fulget Cherub intelligentiae splendore; stat Thronus iudicii firmitate. Igitur si actuosae addicti vitae inferiorum curam recto examine susceperimus, Thronorum stata soliditate firmabimur. $\mathrm{Si}$ ab actionibus feriati, in opificio opificem in opifice opificium meditantes, in contemplandi otio negociabimur, luce cherubica undique corruscabimus. Si caritate ipsum opificem solum ardebimus, illius igne, qui edax est, in saraphicam effigiem repente flammabimur," Garin, p. 110 (souligné par moi).

36. Pic y revient plusieurs fois, cf. Garin, pp. 112-122 passim.

37. "Dabo hoc mihi, et meipsum ex hac parte laudare nihil erubescam, me nunquam alia de causa philosophatum nisi ut philosopharer, nec ex studiis meis, ex meis lucubrationibus, mercedem ullam aut fructum vel sperasse alium vel quaesiisse, quam animi cultum et a me semper plurimum desideratae veritatis cognitionem. Cuius ita cupidus semper et amantissimus fui ut, relicta omni privatarum et publicarum rerum cura, contemplandi otio totum me tradiderim," Garin, p. 132.

38. "Scribis appetere tempus, ut me alicui ex summis Italiae principibus dedam. Adhuc philosophantium de se opinionem non nosti, qui iuxta Horatium se regum reges putant, mores pati, \& servire nesciunt, secum habitant, \& sua contenti animi tranquillitate, sibi ipsis ipsi supersunt, nihil extra se quaerunt; [ . . . Aurea illa optanda mediocritas, qua nos uti man(n)us vehat aequalibus, \& imperii patiens nobis vere serviat non dominetur. In hac ego opinione perstans sellulam meam, mea studia, meorum librorum oblectamenta, meam animi pacem, regiis aulis, publicis negotiis, vestris aucupiis, curiae favoribus, antepono," Opera omnia, fol. 377.

39. Citons encore D. Letocha: "L'esprit moderne a dévalorisé et désinvesti la culture première, sous ses formes institutionnelles et non institutionnelles. À la place, il pose des démarches de construction théorique de l'État, de l'école, de l'armée, de la justice, des réseaux de production industrielle et d'échanges calculés," Ibid., p. 10.

40. Détail caractéristique, Jean-François, son neveu et biographe, note avec étonnement que son oncle se préoccupait si peu de l'état de ses finances qu'il dédaignait même de vérifier les comptes que lui présentait son majordome, sachant qu'il serait bien incapable d'y déceler une fraude; cf. la Vita de Jean Pic, rééditée par Tommaso Sorbelli (Modène, Aedes Muratoria, 1963), p. 72.

41. Cf. Paul Benoît, "Calcul, algèbre et marchandise," dans Éléments d'histoire des sciences, sous la direction de Michel Serres (Paris, Bordas, 1989), pp. 197-221.

42. "Scribit Plato in Epinomide, inter omnes liberales artes et scientias contemplatrices praecipuam maximeque divinam esse scientiam numerandi (...) Quae vera esse nullo modo possunt, si per numerandi artem eam artem intellexerunt cuius nunc mercatores in primis sunt peritissimi, quod et Plato testatur, exserta nos admonens voce ne divinam hanc arithmeticam mercatoriam esse arithmeticam intelligamus," Garin, pp. 146-148.

43. Soit, dans l'ordre, les 47 "Conclusions selon la doctrine des sages Hébreux de la Cabale," les 26 "Conclusions magiques" et les 72 "Conclusions cabalistiques." 
44. Frances A. Yates, "Giovanni Pico della Mirandola and Magic," dans L'Opera e il pensiero di Giovanni Pico della Mirandola nella storia dell'umanesimo(Florence, Istituto Nazionale di Studi sul Rinascimento, 1965), p. 196.

45. En particulier Enn. IV, 4, cc. 35-45.

46. Charles Trinkaus soulignait également que "Pico's image of man is basically a religious image, and also a poetic one, (...) it was not an operative image because it was not an experimental-magical one," In our Image and Likeness: Humanity and Divinity in Italian Humanist Thought (London, Constable, 1970), vol. 2, p. 521.

47. Cf. Giovanni Di Napoli, Giovanni Pico della Mirandola e la problematica dottrinale del suo tempo (Rome, Paris, Tournai, New York, Desclée \& Co., 1965), pp. 211-212.

48. Notons cependant, mais cela ne fait guère partie du présent débat, que Pic se montre "moderne" par l'intériorisation de sa spiritualité, et sans doute aussi par la réorientation qu'il lui donnera. Il ne considère plus que la vie monacale ou conventuelle soit, comme telle, supérieure à la vie laïque. D’où sa résistance aux incitations de Savonarole, et son refus d'entrer dans les ordres. Peut-être peut-on déceler ici l'influence lointaine de la mystique rhénane, mais certainement celle, toute proche et constante, de son fidèle ami Girolamo Benivieni. Sans doute peut-on faire valoir que, par cette intériorisation, Pic aurait en quelque sorte préfiguré l'ère des Réformateurs. Par ailleurs, Jean-François soulignait que, tout en étant parfaitement fidèle aux rites qu'impose l'Église, son oncle " $n$ 'attachait guère de prix aux signes extérieurs de piété"-("exterioris latriae cultus non multum diligens fuerat. Non de eo loquimur quem observandum praecipit ecclesia (gestasse hunc quippe prae oculis eum vidimus) sed de his cerimoniis mentionem facimus, quas nonnulli, posthabito vero cultu Dei, qui in spiritu et veritate colendus est, prosequuntur et provehunt," Vita, p. 74) Revalorisation du laïcat, d'une part, retour à plus de sobriété cultuelle, de l'autre, deux traits qui sans doute présagent le renouveau dont Erasme se fera le porte-parole... A-propos de Jérôme Benivieni, cf. Olga Zorzi Pugliese, "Girolamo Benivieni: umanista riformatore (dalla corrispondenza inedita)," dans La Bibliofilia, LXXII, 1970.

49. J'ai eu l'occasion de développer cette analyse lors du récent Congrès international des Sciences historiques (Montréal 1995), où j'ai présenté une communication intitulée "La condamnation de Jean Pic de la Mirandole: Révvaluation des enjeux". Cette communication paraîtra dans les Actes de la section "Le contrôle des idées à la Renaissance." 